This is the accepted version of the article:

Cappai A., Antidormi A., Bosin A., Narducci D., Colombo L., Melis C.. Impact of synthetic conditions on the anisotropic thermal conductivity of poly (3,4-ethylenedioxythiophene) (PEDOT): A molecular dynamics investigation. Physical Review Materials, (2020). 4. 035401: - .

10.1103/PhysRevMaterials.4.035401.

Available at:

https://dx.doi.org/10.1103/PhysRevMaterials.4.035401 


\title{
Impact of synthetic conditions on the anisotropic thermal conductivity of poly(3,4-ethylenedioxythiophene) (PEDOT): A molecular dynamics investigation
}

\author{
Antonio Cappai $\odot,{ }^{1}$ Aleandro Antidormi $\odot,{ }^{2}$ Andrea Bosin, ${ }^{1}$ Dario Narducci $\odot,{ }^{3}$ Luciano Colombo $\odot,{ }^{1}$ and Claudio Melis $\oplus^{1, *}$ \\ ${ }^{1}$ Department of Physics, University of Cagliari, Cittadella Universitaria, I-09042 Monserrato (CA), Italy \\ ${ }^{2}$ Catalan Institute of Nanoscience and Nanotechnology (ICN2), CSIC and BIST, Campus UAB, Bellaterra, 08193 Barcelona, Spain \\ ${ }^{3}$ Department of Materials Science, University of Milano-Bicocca, via Cozzi 55, 20125 Milano, Italy
}

(Received 23 December 2019; accepted 10 February 2020; published xxxxxx)

\begin{abstract}
In this work we study the effect of different synthetic conditions on thermal transport properties of poly(3,4ethylenedioxythiophene) (PEDOT) by focusing in particular on the role of proton scavengers. To this aim, different PEDOT samples were generated in silico using a novel computational algorithm based on a combination of first-principles density functional theory and classical molecular dynamics simulations. The corresponding thermal conductivities were then estimated using the approach to equilibrium molecular dynamics methodology. The results show that the initial synthetic conditions strongly affect the corresponding thermal conductivities, which display variations up to a factor of $\sim 2$ depending on the proton scavenger. By decomposing the thermal conductivity tensor along the direction of maximum chain alignment and the corresponding perpendicular directions, we attribute the thermal conductivity differences to the variations in the average polymer chain length $\lambda_{\text {ave }}$. A dependence of the thermal conductivity with the polydispersity index was finally observed, suggesting a possible role of intercrystallite chains in enhancing thermal transport properties. By means of the Green-Kubo modal analysis, we eventually characterize the vibrational modes involved in PEDOT thermal transport and investigate how they are related to the thermal conductivity values of the samples.
\end{abstract}

DOI: 10.1103/PhysRevMaterials.00.005400

\section{INTRODUCTION}

In the ongoing quest for nonfossil energy supplies, thermoelectric devices are a promising opportunity. Thermal energy is in fact produced almost ubiquitously, and in large amounts, in all conventional power supply stations, but a very large fraction (usually referred to as waste heat) is almost completely lost. In this scenario, the discovery of efficient thermoelectric materials could promote a sizable replacement of conventional devices for energy production with more environment-friendly thermoelectric ones [1]. This situation has motivated intense experimental and theoretical efforts aimed at increasing the efficiency of thermoelectric generators (TEGs), as well as at searching for new materials with optimal physical features. In this respect, organic conductive polymers are possible candidates as active constituents of micro-TEGs $[2,3]$. In fact, even if in terms of conversion efficiency they are not comparable with their inorganic counterparts, organic polymers are under investigation because of their interesting properties such as flexibility and biocompatibility, very useful in some specific applications where efficiency of conversion is not the main goal. The efficiency of conversion can be quantified by means of the figure of merit $Z T$ [4], defined by a combination of the electrical conductivity $\sigma$, the Seebeck coefficient $S$, and the thermal conductivity $\kappa[5,6]$ as

$$
Z T=\frac{\sigma S^{2}}{\kappa} T
$$

*Corresponding author: cmelis@dsf.unica.it where $T$ is the average operating temperature. As mentioned, at the state of the art, current values of $Z T$ in organic polymers are disappointingly low to make possible large-scale energy production by organic-based TEGs $[3,7,8]$. Hence, several investigations have been devoted to devise new strategies to achieve at least a meaningful increase of $Z T$ [9-12].

It is generally recognized that the thermal and electrical properties of organic polymers are usually strongly dependent on their synthesis and on the resulting morphological features [13]. This state of affairs justifies the large variability of thermal and electrical conductivities reported in the literature. In particular, the chain length has been identified as one of the key factors in determining the overall transport properties [14].

Among organic polymers, poly(3,4-ethylenedioxythiophene) (PEDOT) is especially promising because of its electrical properties (having a hole mobility as high as 3 $\mathrm{cm}^{2} \mathrm{~V}^{-1} \mathrm{~s}^{-1}[15]$ ), air stability [16,17], and low thermal conductivity. According to several experimental and computational investigations, thermal conductivity values have been reported as small as $0.3 \mathrm{~W} \mathrm{~m}^{-1} \mathrm{~K}^{-1}$ for poly(3,4ethylenedioxythiophene):tosylate (PEDOT:tos) samples [18-21]. This good feature is paralleled by a simple synthesis process which allows large-scale manufacturing [16,22].

In a previous work [23] we demonstrated how the polymerization conditions, which are dictated by the choice of proton scavengers, greatly affect the micromorphology of the resulting PEDOT samples, with efficient proton scavengers leading to an increase of the maximum and average chain lengths. Moreover, we analyzed the impact of the chainlength distribution on the degree of crystallinity. However, 
the actual interplay between the synthetic process and the resulting thermoelectric properties is still a matter of debate. Motivated by this, in this paper we explore the impact of the proton scavengers on thermal transport. By combining molecular dynamics simulations and the Green-Kubo modal analysis [24] we compute the main component of thermal conductivity tensor $\stackrel{\leftrightarrow}{\kappa}$. A modal analysis is then performed in order to identify the impact of morphology on the vibrational modes involved.

We provide evidence that PEDOT thermal conductivity is strongly affected by the initial synthetic conditions, i.e., the choice of the proton scavengers employed in the polymerization process. By analyzing the thermal conductivity components along the direction of maximum chain alignment, we attribute such a difference to the variations in the average chain length $\lambda_{\text {ave }}$. Furthermore, by estimating the spatial extension of the different vibrational modes involved in PEDOT thermal transport, we observe large differences depending on the proton scavengers used.

\section{METHOD}

\section{A. Sample preparation}

We simulated the entire polymerization process starting from initial monomers and taking properly into account the influence of different combinations of the main reactants used in real experimental conditions. The computational protocol employed and the stipulated reaction mechanism are described in full detail in our previous work [23]. Here we briefly outline the main features of the two-step algorithm. The Gibbs free energy variation $\Delta G(n)$ (where $n$ is the number of monomeric units composing the oligomer) is first calculated for each polymerization reaction:

$$
\Delta G(n)=\Delta G_{0}(n)+\Delta G(n)^{0 \rightarrow *}+\Delta G(n)_{\text {sol }},
$$

where $\Delta G_{0}(n)$ is the gas-phase Gibbs free energy of reaction

$$
\begin{array}{r}
\mathrm{HX}_{\mathrm{n}-1} \mathrm{H}^{+}+\mathrm{HXH}^{+}+\mathrm{A}^{\mathrm{m}}+\mathrm{B}^{\mathrm{m}^{\prime}} \\
\rightleftharpoons \mathrm{HX}_{\mathrm{n}} \mathrm{H}+\mathrm{AH}^{\mathrm{m}+1}+\mathrm{BH}^{\mathrm{m}^{\prime}+1}
\end{array}
$$

where $\mathrm{HX}_{\mathrm{n}-1} \mathrm{H}$ and $\mathrm{HX}_{\mathrm{n}} \mathrm{H}$ are polymer chains of length $\mathrm{n}-1$ and $\mathrm{n}$, respectively, and $\mathrm{A}^{\mathrm{m}}$ and $\mathrm{B}^{\mathrm{m}^{\prime}}$ are the proton scavengers, either neutral $\left(\mathrm{m}, \mathrm{m}^{\prime}=0\right)$ or anionic $\left(\mathrm{m}, \mathrm{m}^{\prime}=-1\right) . \Delta G^{0 \rightarrow *}$ is the thermodynamic correction accounting for the transition from gas-phase state to standard condition [25], which can be calculated as

$$
\Delta G^{0 \rightarrow *}=\Delta n R T \ln \left(\frac{R T}{p V^{*}}\right),
$$

where $\Delta \mathrm{n}$ is the difference between the sum of moles of products and the sum of moles of reactants, $p$ is the gas-phase pressure ( $1 \mathrm{~atm}$ in the present case), $1 / V^{*}$ is the solute concentration (assumed to be $1 \mathrm{~mol} \mathrm{~L}^{-1}$ ), $T$ the polymerization temperature, and $R$ the ideal gas constant.

Finally, $\Delta G_{\text {sol }}$ is the solvation Gibbs free energy accounting for solute-solvent interactions for each reactant and product. The scavengers investigated in this work are summarized in Table I.

At this stage a fully $a b$ initio calculation is performed via density functional theory (DFT), as implemented in the
TABLE I. Polymerization conditions analyzed in this work and eigenvalues $\varepsilon_{\|}$and $\varepsilon_{p 1, p 2}$ associated with the three axes of the inertia tensor calculated for each sample. Eigenvalues are expressed in nondimensional units.

\begin{tabular}{lccccc}
\hline \hline Sample ID & Scavenger A & Scavenger B & $\varepsilon_{\|}$ & $\varepsilon_{p 1}$ & $\varepsilon_{p 2}$ \\
\hline pyr & Pyridine & Pyridine & 0.82 & 0.76 & 0.42 \\
pyr+wat & Pyridine & Water & 0.81 & 0.70 & 0.49 \\
tos & Tosylate & Tosylate & 0.83 & 0.73 & 0.43 \\
pyr+tos & Pyridine & Tosylate & 0.77 & 0.73 & 0.50 \\
tos+wat & Tosylate & Water & 0.78 & 0.65 & 0.57 \\
wat & Water & Water & 0.70 & 0.66 & 0.64 \\
\hline \hline
\end{tabular}

GAUSSIAN 16 software package [26]. Then, a simulation box containing $N$ 3,4-ethylenedioxythiophene (EDOT) monomers is generated and aged in an NVT ensemble by means of classical molecular dynamics, using the LAMMPS software package [27]. Interactions have been described using the AMBER force field [28]. A cutoff radius of $10 \AA$ is used for Lennard-Jones interactions, while electrostatic long-range interactions (i.e., exceeding the cutoff radius) were computed using a particleparticle particle-mesh (pppm) solver with an accuracy of $10^{-4}$ kcal mol ${ }^{-1} \AA^{-1}$.

The formation of a chemical bond between two molecules is described as a Markov-like mechanism: using the $\Delta G(n)$ calculated in the previous step, a bond creation probability $p(n)$ is defined as

$$
p(n)=\min \left(1, \exp \left(\Delta G(n) /\left(k_{B} T\right)\right)\right),
$$

where $k_{B}$ is the Boltzmann constant and $T$ the operating temperature, to quantitatively describe how likely is the formation of a bond between two units closer than a given distance threshold ( $3 \AA$ in our case). If the bond is created, the force field underlying molecular dynamics (MD) simulations is updated by introducing the new bonds, angles, and dihedrals using an approach similar to the methodology developed by Gissinger et al. [29]. By developing a specific polymerization code applying this protocol, PEDOT samples resulted to be characterized by different chain-length distributions. The average chain length ranged from 2 up to 19 monomeric units.

In order to evaluate the impact of the chain-length distribution on thermal transport, the protocol described in the next section has been used.

\section{B. Thermal conduction}

The calculation of thermal conductivity in our samples was performed using the approach to equilibrium molecular dynamics (AEMD) method [30-32]: a steplike temperature profile was initially imposed within the system along a given direction, next evolving according to the heat equation

$$
\rho c \frac{\partial T}{\partial t}=\vec{\nabla} \cdot(\overleftrightarrow{\kappa} \cdot \vec{\nabla} T)
$$

where $\overleftrightarrow{\kappa}$ is the thermal conductivity tensor, $\rho$ the mass density, and $c$ the specific heat.

In order to create the steplike profile, two regions, one for each half of the sample, were defined and, respectively, equilibrated at the target temperature $T_{1}=350 \mathrm{~K}$ and $T_{2}=$ 


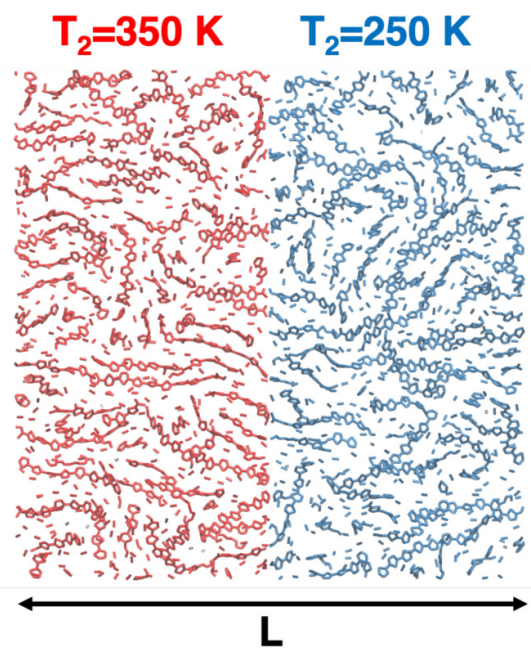

FIG. 1. Simulation box for AEMD simulation. The simulations cell ( $L$ side) is divided in a hot region (left, red), thermostatted at $T_{1}=350 \mathrm{~K}$, and a cold region (right, blue) at $T_{2}=250 \mathrm{~K}$ to establish a steplike temperature profile.

$250 \mathrm{~K}$ using a Nosé-Hoover thermostat (Fig. 1). During the equilibration of the left (right) region, the atoms of the opposite right (left) region were kept fixed in order to avoid any heat exchange during the thermostatting period. After the initial equilibration, the following unconstrained dynamics was generated in an $N V E$ ensemble by recording the average temperatures $\left\langle T_{1}^{i}(t)\right\rangle$ and $\left\langle T_{2}^{i}(t)\right\rangle$ of both regions. Since the samples display no structural order, the initial temperature profile was separately imposed along the $i=x, y, z$ directions, corresponding to the orthogonal edges of the periodically repeated cubic simulation box ( $L$ side).

The evolution of the average temperature difference $\Delta T^{i}(t)=\left\langle T_{2}^{i}(t)\right\rangle-\left\langle T_{1}^{i}(t)\right\rangle$ between the two regions of the system was fitted onto the solution of Eq. (6), which reads

$$
\Delta T^{i}(t)=\sum_{n=0}^{+\infty} \frac{8 \Delta T^{i}(0)}{\pi^{2}(2 n+1)^{2}} \exp \left(-\frac{\kappa_{i i} \pi^{2}}{\rho c L^{2}}(2 n+1)^{2} t\right),
$$

by using the fact that off-diagonal components of the thermal conductivity tensor $\overleftrightarrow{\boldsymbol{\kappa}}$ were found to be sufficiently small to be negligible. Because of this, only diagonal components $\kappa_{i i}$ enter in Eq. (7). We remark that in the fitting procedure the sum was truncated at $n=20$. We checked that further increases of $n$ do not affect $\kappa_{i i}$.

Simulations were carried out on cubic samples of $118 \AA$ side in periodic boundary conditions along each direction: each sample contains as many as $\sim 10^{5}$ atoms. Equations of motion have been integrated by the velocity Verlet algorithm with a time step of $0.5 \mathrm{fs}$. Since the temperature-difference profiles appear to be very noisy when the average temperature difference between the regions is below $\sim 5 \mathrm{~K}$, an ensemble average (ten trajectories for each curve) was performed for each sample by varying the initial equilibration periods.

\section{Modal analysis}

In order to unveil the effect of the chain-length distribution on the thermal properties of the samples, the Green-Kubo modal analysis (GKMA) was employed [33]. Relying both on long MD runs and on the diagonalization of the dynamical matrix [34], this methodology represents a valuable tool to investigate thermal properties in systems with broken translation invariance, where the validity of concepts like phononic group velocity and mean free paths is questionable. For this reason, GKMA has already been successfully employed in the study of amorphous and paracrystalline materials [33].

In GKMA, each Cartesian component $\kappa_{\mu \nu}$ of the thermal conductivity tensor $\overleftrightarrow{\boldsymbol{\kappa}}$ is calculated from the equilibrium current-current autocorrelation function, according the GreenKubo relation

$$
\kappa_{\mu \nu}=\frac{\Omega}{k_{B} T^{2}} \int_{0}^{\infty}\left\langle q_{\mu}(t) \cdot q_{\nu}(0)\right\rangle d t,
$$

where $\Omega$ is the system volume, $k_{B}$ is the Boltzmann constant, $T$ is the system temperature, and the angular brackets denote an ensemble average of the product of the heat fluxes $\mu, v$ Cartesian components $q_{\mu, \nu}(t)$ at a given time $t$. The components are obtained from the vibrational eigenmodes extracted from the dynamical matrix, defined as [33]

$$
D_{l \alpha, j \beta}=-\frac{1}{\sqrt{m_{l} m_{j}}} \frac{\partial F_{l \alpha}}{\partial r_{j \beta}},
$$

where $m_{l}$ is the mass of the $l$ th atom, $F_{l \alpha}$ the $\alpha$ Cartesian component of the force acting on the $l$ th atom, and $r_{j \beta}$ the $\beta$ Cartesian component of the displacement of the $j$ th atom.

The eigenmodes of the system, extracted from the diagonalization of the dynamical matrix given in Eq. (9), yield a set $\mathbf{e}_{l, s}$ of $3 N$ vectors (with $N$ the number of atoms in the sample). For each $s$ eigenmode, a heat flux operator $\mathbf{Q}_{s}(t)$ is defined according to

$$
\mathbf{Q}_{s}(t)=\frac{1}{\Omega} \sum_{l=1}^{N}\left[E_{l} \dot{\mathbf{x}}_{l}(s, t)+\sum_{k=1}^{N}\left(\mathbf{F}_{l k} \dot{\mathbf{x}}_{l}(s, t) \mathbf{r}_{l k}\right)\right],
$$

where $\Omega$ is the total volume of the sample, $E_{l}$ is the total energy of the $l$ th atom, $\mathbf{F}_{l k}$ is the force acting on the $l$ th atom when the $k$ th atom is displaced, and $\mathbf{r}_{l k}$ is the distance vector between the $l$ th and $k$ th atoms. Each Cartesian component $\mu$ of the heat flux operator $\mathrm{Q}_{\mu, s}(t)$ is related to the corresponding $q_{\mu}(t)$ heat flux in Eq. (8) by the relation

$$
q_{\mu}(t)=\sum_{s=1}^{3 N} \mathrm{Q}_{\mu, s}(t) .
$$

The contribution of the $s$ th mode to the velocity of the $l$ th atom is represented by $\dot{\mathbf{x}}_{l}(s, t)$ and it is obtained by projecting the total velocity $\mathbf{v}_{l}$ of the $l$ th atom onto the eigenvectors of the matrix given in Eq. (9) according to

$$
\mathbf{x}_{l}(s, t)=\left(\mathbf{v}_{l} \cdot \mathbf{e}_{l, s}\right) \mathbf{e}_{l, s} .
$$

During an $N V E$ run, $\mathbf{Q}_{s}(t)$ is calculated for a simulation time as long as $200 \mathrm{ps}$; the overall thermal conductivity $\kappa$, defined (as is common in the literature [33]) in terms of the trace of the thermal conductivity tensor,

$$
\kappa=\frac{1}{3} \operatorname{Tr}(\stackrel{\leftrightarrow}{\kappa})=\frac{\kappa_{x x}+\kappa_{y y}+\kappa_{z z}}{3},
$$



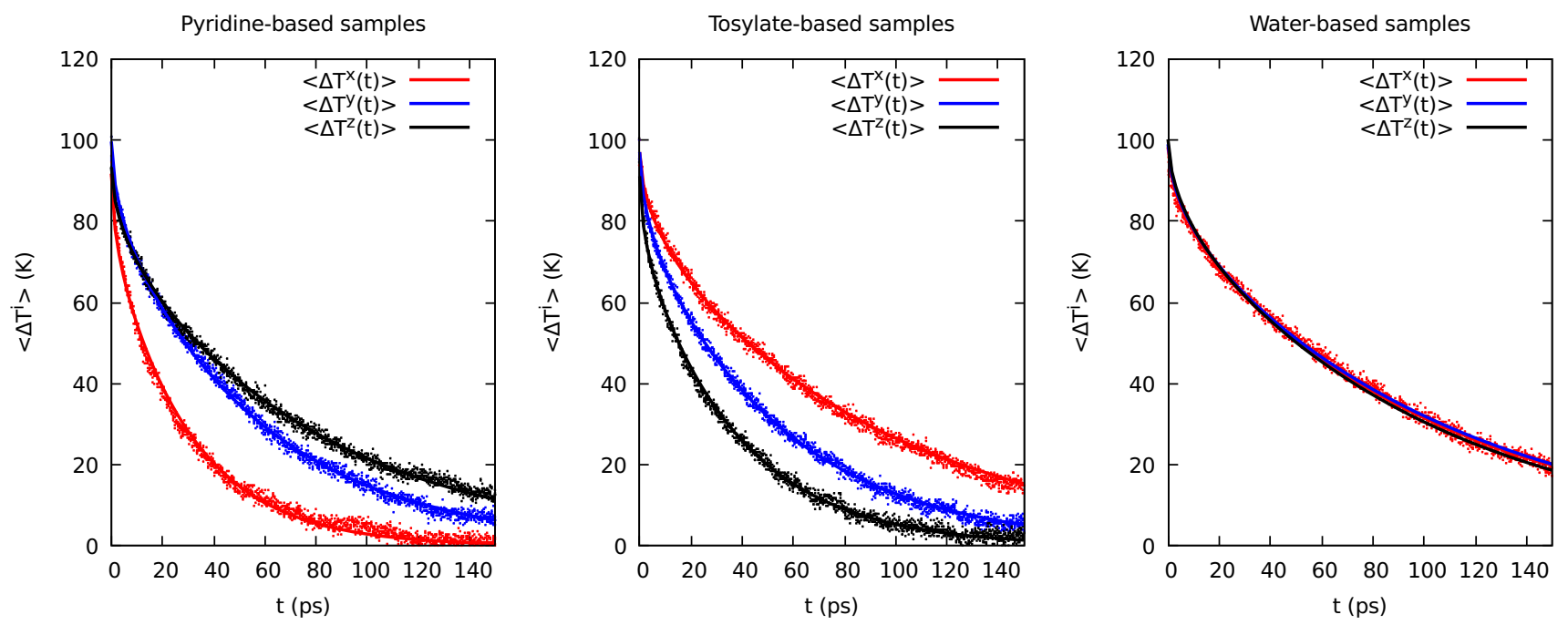

FIG. 2. Time evolution of the average $\left\langle\Delta T^{i}(t)\right\rangle$ along the three directions $i=x, y, z$ of a pyr (left), tos (center), and wat sample (right): smooth lines represent the result of the fitting on simulated data.

can then be computed within the the Green-Kubo formalism, referring to Eqs. (8) and (11), as

$$
\kappa=\frac{\Omega}{3 k_{B} T^{2}} \int_{0}^{\infty}\left\langle\sum_{s=1}^{3 N} \mathbf{Q}_{s}(t) \cdot \sum_{s^{\prime}=1}^{3 N} \mathbf{Q}_{s^{\prime}}(0)\right\rangle d t .
$$

The contribution of each mode can be then explicitly calculated by relying on the total heat flux operator in Eq. (11) evaluated at $t=0$ in Eq. (14), which can be recast in the form

$$
\kappa=\frac{\Omega}{3 k_{B} T^{2}} \sum_{s=1}^{3 N} \int_{0}^{\infty}\left\langle\mathbf{Q}_{s}(t) \cdot \mathbf{q}(0)\right\rangle d t=\sum_{s=1}^{3 N} \kappa_{s},
$$

where $\kappa_{s}$ is the contribution of the $s$ th mode to the thermal conductivity.

In order to investigate the number of monomers involved in each vibrational sth mode, a participation ratio (PR) was defined as

$$
\mathrm{PR}=\frac{1}{N} \frac{\left(\sum_{l=1}^{N} \mathbf{e}_{l, s}^{2}\right)^{2}}{\sum_{l=1}^{N} \mathbf{e}_{l, s}^{4}}
$$

where $\mathbf{e}_{l, s}$ are derived from the diagonalization of the dynamical matrix.

The definition of the PR makes it possible to determine the spatial extension of each of the $s$ modes involved in thermal transport. In fact, if the eigenmode analyzed involves a large group of atoms, i.e., if the spatial extension of the eigenmode is large, $P R \sim 1$, while in the case of localized modes the PR is very close to zero and decays as a function of the number of atoms, $N$, involved [PR $=O\left(N^{-1}\right)$ ] [35]. Starting from this different behavior, Allen et al. [35] proposed, by studying the paradigmatic case of amorphous silicon, a classification of extended modes in diffusons, for which the PR quickly decays to zero, and propagons, characterized by a larger and almost $N$-insensitive value of PR.

Finally, in order to better characterize the vibrational modes, we define [33] the average coordination number as the weighted number of bonds with first neighbors involved in a given mode. It is calculated as

$$
\bar{n}_{s}=\frac{\sum_{l=1}^{N} n_{C, l} e_{l, s}^{2}}{\sum_{l=1}^{N} e_{l, s}^{2}},
$$

where $n_{C, l}$ is the coordination number of the $l$ th atom, and $e_{l, s}^{2} \quad{ }_{267}$ is the squared modulus of its displacement vector.

\section{RESULTS AND DISCUSSIONS}

\section{A. Dependence of thermal conductivity $\kappa$ from the adopted scavenger}

Figure 2 shows the time evolution of the average temperature difference $\left\langle\Delta T^{i}(t)\right\rangle$ during the simulation in the case of pyr (left), tos (center), and wat (right) samples.

For pyr and tos samples, a different $\left\langle\Delta T^{i}(t)\right\rangle$ evolution depending on the $i$ direction is observed, while in the case of wat samples the evolution is isotropic. We argue that the actual choice of the proton scavenger for the polymerization process largely affects the heat propagation, thus generating anisotropy which we attributed to the fact that pyr and tos samples shows significantly higher crystalline volume fractions with respect to wat samples [23], as well as higher average chain lengths $\lambda_{\text {ave }}$.

In order to better clarify the observed anisotropies occurring for relatively high $\lambda_{\text {ave }}$ values, we estimated the components of the thermal conductivity tensor $\overleftrightarrow{\boldsymbol{\kappa}}$ along the direction of maximum chain alignment $\left(\kappa_{\|}\right.$component) and along the two corresponding orthogonal directions ( $\kappa_{p 1, p 2}$ components). They were obtained as follows.

Starting from a polymerized sample, we defined an orientation vector for each unit as the direction identified by the position of two specific carbon atoms of the thiophenic ring [Fig. 3(a)]. These $M$ normalized vectors were then translated in order to point out from a specific origin [Fig. 3(b)]. This representation is equivalent to $M$ points within a sphere [yellow in Fig. 3(b)] of unitary radius. In systems characterized by long-range order, it is expected to observe all such points concentrated around two poles of the sphere, corresponding 


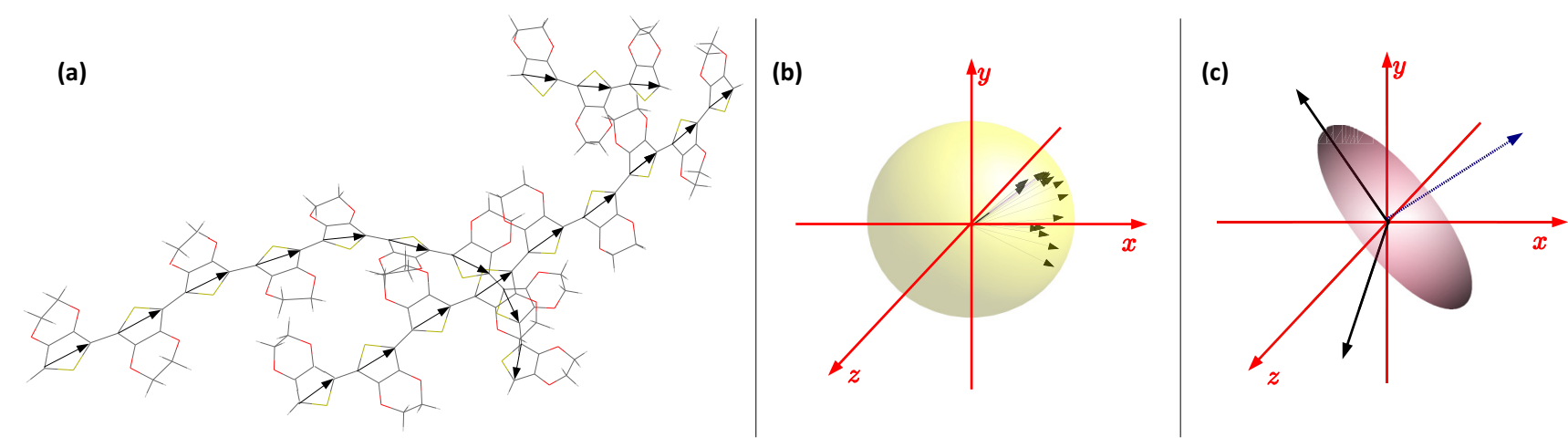

FIG. 3. Schematic representation of the procedure used to estimate the direction of maximum chain alignment: (a) polymeric configuration in real space: the black arrows represents the specific $\mathrm{C}-\mathrm{C}$ orientational vectors; (b) polymeric configuration in the orientational vector space where unitary fictitious masses are attributed to each point lying on the unitary sphere surface; and (c) representation of the inertia tensor in the orientational vector space: the blue arrow represents the direction of maximum alignment.

to the direction of maximum chain alignment. In amorphous structure, instead, the distributions of these points within the sphere must be uniform, since no special directions are present in the sample.

In order to determine the direction along which the largest number of chains is aligned, a fictional unitary mass was attributed to each of the $M$ points and all the components of the corresponding inertia tensor were computed. The mean direction of maximum alignment can then be found by estimating the eigenvalues of the real non-negative corresponding density matrix and looking for its column vector associated with the lowest eigenvalue [Fig. 3(c)].

The analysis of the eigenvalues (Table I) associated with each density map clearly suggests the existence of a longrange order in pyr samples. At variance, wat samples appear to be mainly amorphous. This observation is consistent with the results reported in Fig. 2 and previously [23], where we provided evidence about the increase in crystallinity of pyr samples compared to wat samples.

After the calculations of the three axes of the inertia ellipsoid, the inertia and thermal conductivity tensors were simultaneously diagonalized in order to obtain the thermal

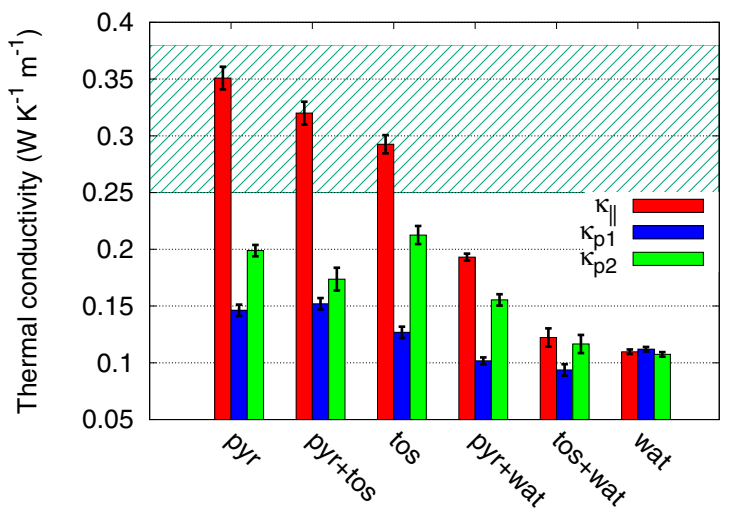

FIG. 4. Thermal conductivity components along the direction of maximum alignment, $\kappa_{\|}$, and the two orthogonal directions, $\kappa_{p 1, p 2}$. The dashed band displays the range of experimental $\kappa$ previously reported for PEDOT:tos samples [18-21]. conductivity component along these three main directions. The off-diagonal entries resulted in being negligible with respect to the diagonal $\kappa_{\|}, \kappa_{p 1}, \kappa_{p 2}$ components, which are plotted for each sample in Fig. 4. The dashed band represents the $\kappa$ previously reported for PEDOT:tos samples [18-21].

The results provide robust evidence of the role of chain length in determining the magnitude of the resulting thermal conductivity: it is in fact shown that samples characterized by longer chains (pyr and pyr+tos samples) exhibit higher in-chain thermal conductivities compared to wat samples, in which $\kappa_{\|}$reaches its minimum, resulting in being indistinguishable from the two $\kappa_{p 1}, \kappa_{p 2}$ components.

Referring to Fig. 4, we observed a good agreement between the results for $\kappa_{\|}$obtained from our calculations, $\kappa_{\|}=(0.29 \pm$ $0.01) \mathrm{Wm}^{-1} \mathrm{~K}^{-1}$, and the values reported in the literature [21], $\kappa_{\|}=(0.286 \pm 0.001) \mathrm{Wm}^{-1} \mathrm{~K}^{-1}$, for PEDOT:tos samples.

It is interesting to observe that even if out-of-chain components are negligible with respect to the $\kappa_{\|}$component in high anisotropic samples, a slight reduction is observed moving from pyr samples to the wat ones. We argue that in amorphouslike samples the average distance between chains is greater than the corresponding distance in more crystalline samples, resulting in a decrease of the interaction which,

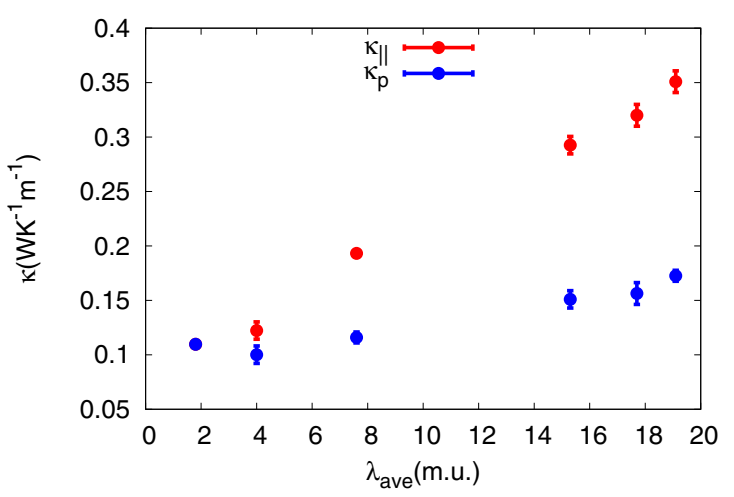

FIG. 5. Average $\kappa_{\|}$and $\kappa_{p}$ values of the samples analyzed plotted as a function of average chain length $\lambda$ (in monomeric units): $\kappa_{p}$ is defined as the average value of $\kappa_{p 1}$ and $\kappa_{p 2}$. 


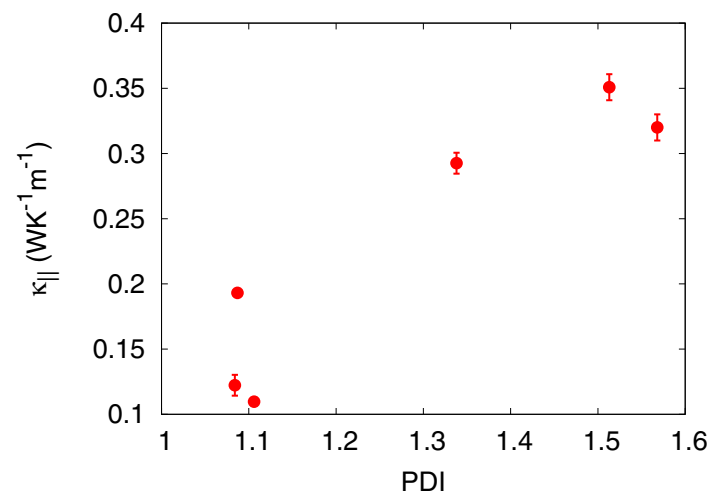

FIG. 6. Average $\kappa_{\|}$values of the samples analyzed plotted as a function of PDI.

in turn, is detrimental for thermal transport. As observed in Ref. [23] an increase in crystallite fraction from $0.8 \%$ to $49 \%$ is related to a shift of the peaks of the sulfur-sulfur radial distribution function of $(0.06 \pm 0.01) \mathrm{nm}$, suggesting a reduction of the average interchain distance.

Figure 5 shows the average $\kappa_{\|}$and $\kappa_{p}$ values of the analyzed samples as a function of $\lambda_{\text {ave }}$. A sharp increase of $\kappa_{\|}$as a function of the average chain length can be observed while the average $\kappa_{p}$ shows a much weaker dependence on $\lambda_{\text {ave }}$. We further observe that samples characterized by longer chains (pyr and pyr+tos samples) exhibit higher parallel thermal conductivities compared to wat samples, in which $\kappa_{\|}$reaches its minimum, resulting in being indistinguishable from the two perpendicular components.

In addition, $\kappa_{\|}$depends also on the polydispersity index (PDI) (Fig. 6), suggesting a possible role for the intercrystallite chain in enhancing the thermal transport properties of PEDOT, in strict analogy with the effects on electronic transport observed by Noriega et al. [14] and Kang and Snyder [36] in their works.

The strong dependence of thermal conductivity on the chain length is finally confirmed by estimating the thermal conductivity for different samples corresponding to different stages of the polymerization procedure (in the case of pyr samples), in which $\lambda_{\text {ave }}$ increases with time. The result, shown in Fig. 7, clearly shows the impact of increasing the chain

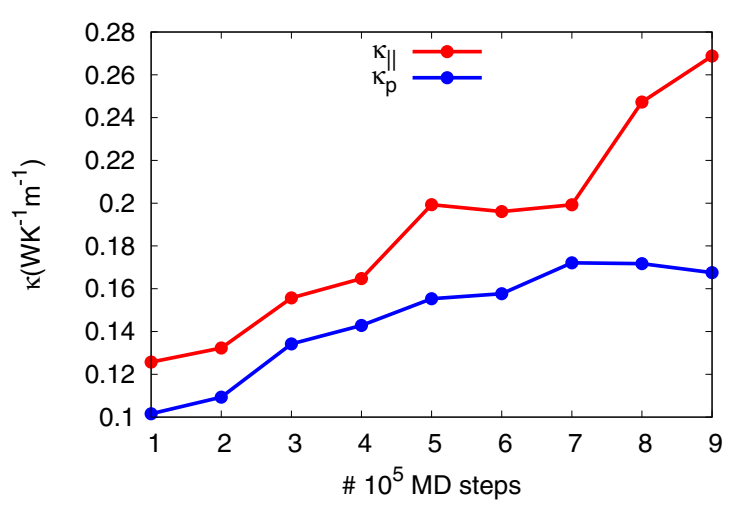

FIG. 7. Evolution of thermal conductivity components of the pyr sample, $\kappa_{\|}$and $\kappa_{p}$, during the polymerization process.

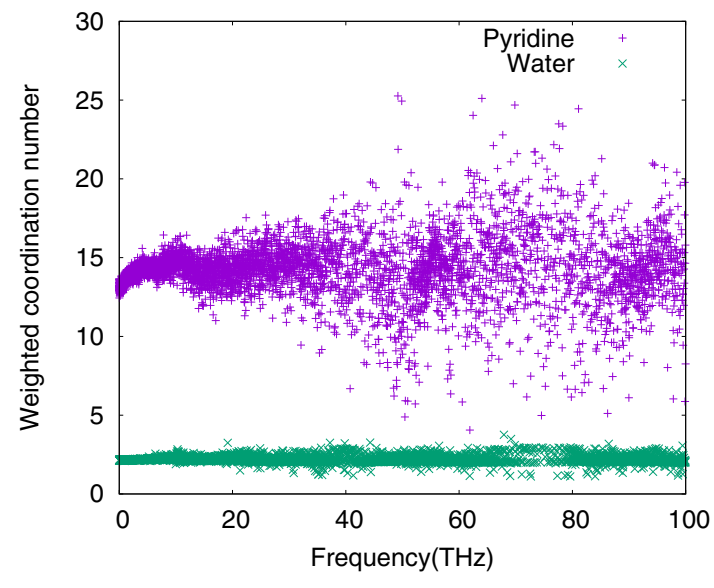

FIG. 8. Average monomeric units involved in vibrational modes as a function of the oscillation frequency for wat (purple) and pyr (green) samples.

length on the overall thermal conductivity. In particular, along the direction of maximum alignment number the increase of $\kappa$ is almost monotonic.

\section{B. Modal analysis results}

In order to provide additional characterization of the impact of morphological features on the vibrational modes (i.e., heat carriers) involved, we performed a Green-Kubo modal analysis. In Fig. 8 the average weighted coordination number $n_{s}$ [see Eq. (17)] for pyr and wat samples is plotted as a function of the frequency $v$ of the vibrational eigenmodes.

Simulations indicate that the number of monomers involved in each vibrational mode in pyr samples is significantly higher compared to that of wat samples. This is quite relevant in explaining the higher thermal conductivity corresponding to pyr samples, since the number of units involved in each vibrational mode is a direct measure of the spatial extent of the mode. We conclude that in pyr samples even low-frequency vibrational modes are characterized by an overall extension of about 12-15 monomeric units. Therefore, they are more effective in the thermal energy propagation if compared to the case of wat samples, in which the extent of each vibrational

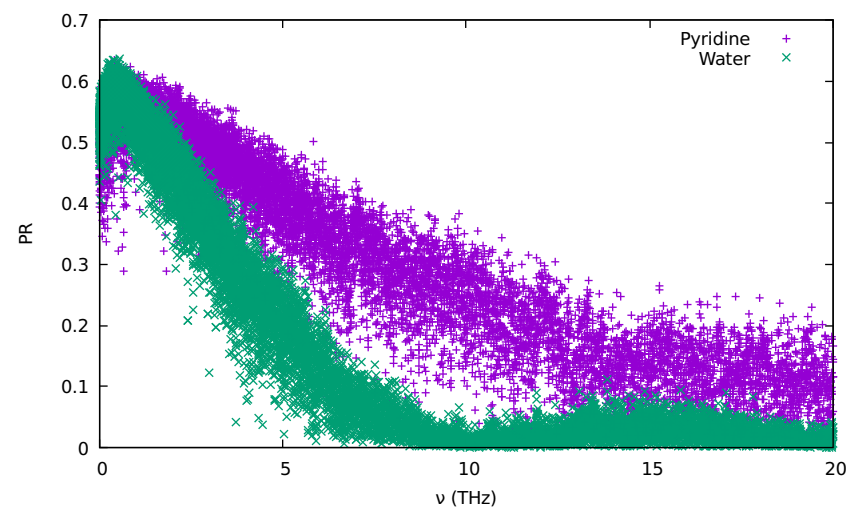

FIG. 9. Estimated PR as a function of the frequency for wat (purple) and pyr (green) PEDOT samples. 


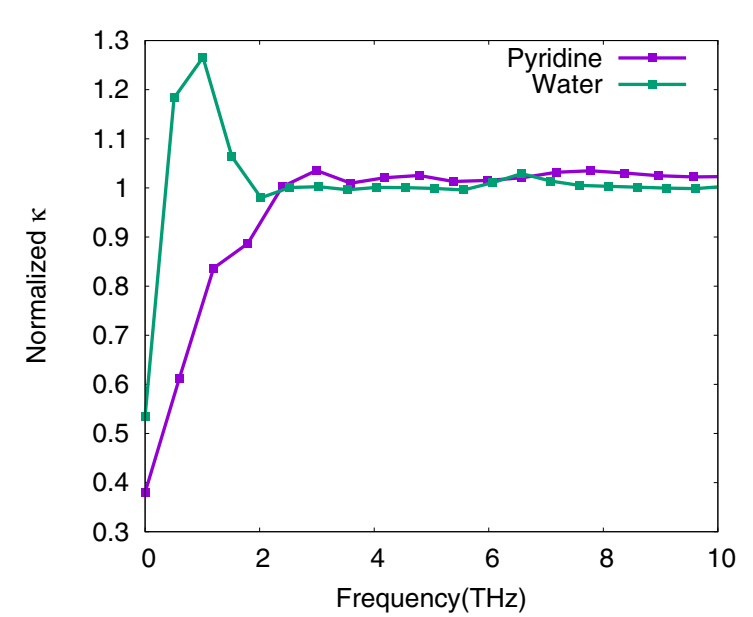

FIG. 10. Normalized cumulative conductivity as function of the frequency for wat (purple) and pyr (green) samples.

mode is limited to two to three monomeric units in all the range of frequencies investigated.

By further analyzing the corresponding participation ratio as defined in Eq. (16) and reported in Fig. 9, we observe that in pyr samples the low-frequency modes are more spatially extended compared to the case of pure wat samples. The participation ratio decay is in fact much slower than in the case of wat samples, determining a larger efficiency in propagating thermal energy. In the case of wat samples, on the other hand, the faster decay of PR indicates that such vibrational eigenmodes are spatially more confined. This property is detrimental in terms of thermal transport since eigenmodes above the frequency threshold of $\sim 5 \mathrm{THz}$ cannot efficiently sustain extended heat currents along all the sample (Fig. 10).

Our picture is confirmed by Fig. 10 in which the normalized cumulative thermal conductivity $\kappa_{s}$ [defined as in Eq. (15)] is shown as a function of the frequency $v$ of the vibrational modes in the range $0-10 \mathrm{THz}$. In both cases depicted in Fig. 10 the cumulative thermal conductivity quickly converges to its final value at around $2 \mathrm{THz}$; however, the approach is different: while in the case of pyr samples the convergence value is reached almost monotonically, in the case of wat samples, after an initial sharp increase, negative contributions are present in the range $1-2 \mathrm{THz}$, leading to a decrease of the cumulative thermal conductivity. This behavior is not in contrast with the definition of $\kappa_{S}(v)$ since the terms involved in Eq. (15) can also be nonpositive, revealing the presence of a sharp decrease in the number of modes involved in heat propagation (the so-called propagons: low-frequency modes extending for several monomeric units before scattering $[33,35,37])$. At the same time, we observe a corresponding increase in the number of modes related with a pure diffusion of the thermal energy (i.e., diffusons: vibrational modes immediately scattered and lacking a meaningful mean free path $[33,35,37])$ for which the participation ratio PR quickly decays [see Eq. (16)].

\section{CONCLUSIONS}

In this work we have investigated the effect of different synthetic procedures on PEDOT thermal transport properties. To this aim we generated realistic PEDOT samples by means of a novel computational algorithm based on a combination of first-principles density functional theory and classical molecular dynamics simulations. We then estimated their corresponding thermal conductivities by using the AEMD methodology.

We observe a clean dependence of PEDOT thermal transport properties on the initial synthetic conditions, i.e., the choice of the proton scavengers employed in the polymerization process. By decomposing the thermal conductivity parallel and perpendicular components with respect to the direction of maximum chain alignment, we prove that the main contribution to the overall $\kappa$ is in the chain direction, while thermal conductivities in the perpendicular directions are significantly lower and less influenced by the average chain length.

We further characterized the vibrational modes involved in PEDOT thermal transport in terms of their spatial extension by computing the average number of monomers involved in each vibrational eigenmode and the contributions of each mode to the thermal conductivity. We remark that, while in the case of pyr samples the spatial extension of the modes is relatively high even at low frequencies $(0-2 \mathrm{THz})$, wat samples are characterized by vibrational modes involving few monomeric units (two or three). Such a difference can be directly related to the different thermal conductivity values: in particular the higher thermal conductivity value estimated for pyr samples is directly caused by the higher chain lengths resulting in a higher delocalization of the corresponding vibrational modes.

The present scenario unveils the critical influence of proton scavengers in determining a high degree of anisotropy in PEDOT thermal transport by affecting the overall average chain length and leading to a spatial limitation of low-frequency vibrational modes crucially responsible for thermal conductivity.

\section{ACKNOWLEDGMENT}

A.C. gratefully acknowledges Regione Autonoma della Sardegna for financial support under project "Programma Operativo Regionale FSE 2014-2020”.
[1] J. D. König, K. Bartholomé, H. Böttner, D. Jänsch, M. Klein Altstedde, M. Köhne, J. Nurnus, A. Roch, K. Tarantik, and G. Hirn, BINE-Themeninfo I/2016, 1 (2016).

[2] J. L. Blackburn, A. J. Ferguson, C. Cho, and J. C. Grunlan, Adv. Mater. 30, 1704386 (2018).
[3] D. Beretta, N. Neophytou, J. M. Hodges, M. G. Kanatzidis, D. Narducci, M. Martin-Gonzalez, M. Beekman, B. Balke, G. Cerretti, W. Tremel et al., Mater. Sci. Eng. R 138, 210 (2019).

[4] M. V. Vedernikov and E. K. Iordanishvili, in 17th International Conference on Thermoelectrics: Proceedings 
ICT98, Nagoya, Japan (IEEE, New York, 1998), pp. $37-42$.

[5] Z. U. Khan, O. Bubnova, M. J. Jafari, R. Brooke, X. Liu, R. Gabrielsson, T. Ederth, D. R. Evans, J. W. Andreasen, M. Fahlman et al., J. Mater. Chem. C 3, 10616 (2015).

[6] K. Wijeratne, M. Vagin, R. Brooke, and X. Crispin, J. Mater. Chem. A 5, 19619 (2017).

[7] C. B. Vining, Nat. Mater. 8, 83 (2009).

[8] A. Shakouri, Annu. Rev. Mater. Res. 41, 399 (2011).

[9] D. A. Mengistie, C.-H. Chen, K. M. Boopathi, F. W. Pranoto, L.J. Li, and C.-W. Chu, ACS Appl. Mater. Interfaces 7, 94 (2015).

[10] F. Kong, C. Liu, H. Song, J. Xu, Y. Huang, H. Zhu, and J. Wang, Synth. Met. 185, 31 (2013).

[11] Z. Zhu, C. Liu, F. Jiang, J. Xu, and E. Liu, Synth. Met. 225, 31 (2017).

[12] M. Culebras, C. Gómez, and A. Cantarero, J. Mater. Chem. A 2, 10109 (2014).

[13] A. Crnjar, C. Melis, and L. Colombo, Phys. Rev. Mater. 2, 015603 (2018).

[14] R. Noriega, J. Rivnay, K. Vandewal, F. P. Koch, N. Stingelin, P. Smith, M. F. Toney, and A. Salleo, Nat. Mater. 12, 1038 (2013).

[15] M. Neophytou, J. Griffiths, J. Fraser, M. Kirkus, H. Chen, C. B. Nielsen, and I. McCulloch, J. Mater. Chem. C 5, 4940 (2017).

[16] A. Elschner, S. Kirchmeyer, W. Lovenich, U. Merker, and K. Reuter, PEDOT: Principles and Applications of an Intrinsically Conductive Polymer (CRC Press, Boca Raton, FL, 2010).

[17] K. Sun, S. Zhang, P. Li, Y. Xia, X. Zhang, D. Du, F. H. Isikgor, and J. Ouyang, J. Mater. Sci.: Mater. Electron. 26, 4438 (2015).

[18] L. Cigarini, A. Ruini, A. Catellani, and A. Calzolari, Phys. Chem. Chem. Phys. 20, 5021 (2018).

[19] O. Bubnova, Z. U. Khan, A. Malti, S. Braun, M. Fahlman, M. Berggren, and X. Crispin, Nat. Mater. 10, 429 (2011).

[20] H. Kwok, J. Electron. Mater. 42, 355 (2013).

[21] K. Zhang, J. Qiu, and S. Wang, Nanoscale 8, 8033 (2016).

[22] W. Hong, Y. Xu, G. Lu, C. Li, and G. Shi, Electrochem. Commun. 10, 1555 (2008).

[23] A. Cappai, A. Antidormi, A. Bosin, D. Galliani, D. Narducci, and C. Melis, Phys. Chem. Chem. Phys. 21, 8580 (2019).

[24] H. R. Seyf, K. Gordiz, F. DeAngelis, and A. Henry, J. Appl. Phys. 125, 081101 (2019).

[25] G. C. Shields and P. G. Seybold, Computational Approaches for the Prediction of $p K_{a}$ Values (CRC Press, Boca Raton, FL, 2013).
[26] M. J. Frisch, G. W. Trucks, H. B. Schlegel, G. E. Scuseria, M. A. Robb, J. R. Cheeseman, G. Scalmani, V. Barone, G. A. Petersson, H. Nakatsuji, X. Li, M. Caricato, A. V. Marenich, J. Bloino, B. G. Janesko, R. Gomperts, B. Mennucci, H. P. Hratchian, J. V. Ortiz, A. F. Izmaylov, J. L. Sonnenberg, D. Williams-Young, F. Ding, F. Lipparini, F. Egidi, J. Goings, B. Peng, A. Petrone, T. Henderson, D. Ranasinghe, V. G. Zakrzewski, J. Gao, N. Rega, G. Zheng, W. Liang, M. Hada, M. Ehara, K. Toyota, R. Fukuda, J. Hasegawa, M. Ishida, T. Nakajima, Y. Honda, O. Kitao, H. Nakai, T. Vreven, K. Throssell, J. A. Montgomery, Jr., J. E. Peralta, F. Ogliaro, M. J. Bearpark, J. J. Heyd, E. N. Brothers, K. N. Kudin, V. N. Staroverov, T. A. Keith, R. Kobayashi, J. Normand, K. Raghavachari, A. P. Rendell, J. C. Burant, S. S. Iyengar, J. Tomasi, M. Cossi, J. M. Millam, M. Klene, C. Adamo, R. Cammi, J. W. Ochterski, R. L. Martin, K. Morokuma, O. Farkas, J. B. Foresman, and D. J. Fox, GAUSSIAN16 Revision C.01 (gaussian Inc., Wallingford, CT, 2016).

[27] S. Plimpton, J. Comput. Phys. 117, 1 (1995).

[28] D. A. Case, I. Y. Ben-Shalom, S. R. Brozell, D. S. Cerutti, T. E. Cheatham, III, V. W. D. Cruzeiro, T. A. Darden, R. E. Duke, D. Ghoreishi, M. K. Gilson, H. Gohlke, A. W. Goetz, D. Greene, R. Harris, N. Homeyer, S. Izadi, A. Kovalenko, T. Kurtzman, T. S. Lee, S. LeGrand, P. Li, C. Lin, J. Liu, T. Luchko, R. Luo, D. J. Mermelstein, K. M. Merz, Y. Miao, G. Monard, C. Nguyen, H. Nguyen, I. Omelyan, A. Onufriev, F. Pan, R. Qi, D. R. Roe, A. Roitberg, C. Sagui, S. Schott-Verdugo, J. Shen, C. L. Simmerling, J. Smith, R. Salomon-Ferrer, J. Swails, R. C. Walker, J. Wang, H. Wei, R. M. Wolf, X. Wu, L. Xiao, D. M. York, and P. A. Kollman, AMBER (University of California, San Francisco, 2018).

[29] J. R. Gissinger, B. D. Jensen, and K. E. Wise, Polymer 128, 211 (2017).

[30] E. Lampin, P. L. Palla, P.-A. Francioso, and F. Cleri, J. Appl. Phys. 114, 033525 (2013).

[31] C. Melis, R. Dettori, S. Vandermeulen, and L. Colombo, Eur. Phys. J. B 87, 96 (2014).

[32] G. Fugallo and L. Colombo, Phys. Scr. 93, 043002 (2018).

[33] A. Antidormi, X. Cartoixà, and L. Colombo, Phys. Rev. Mater. 2, 056001 (2018).

[34] W. Lv and A. Henry, New J. Phys. 18, 013028 (2016).

[35] P. B. Allen, J. L. Feldman, J. Fabian, and F. Wooten, Philos. Mag. B 79, 1715 (1999).

[36] S. Dongmin Kang and G. Snyder, Nat. Mater. 16, 252 (2017).

[37] H. R. Seyf and A. Henry, J. Appl. Phys. 120, 025101 (2016). 